\title{
Steroid Induced Bilateral Femoral Head Avascular Necrosis in a Patient of Minimal Change Glomerulonephritis: Detection by Radionuclide Bone Scan
}

\author{
Shoukat Hussain Khan, M.D., ${ }^{1}$ Tanveer Ahmed Rather, M.D., 'Manjeet Singh, M.D. ${ }^{2}$ \\ 'Departments of Nuclear Medicine, and '2Radiology, Sher-i-Kashmir Institute of Medical Sciences, Srinagar, J \&K, India- 190011
}

\section{A B S T R A C T}

One of the commonest causes of avascular necrosis of bones in patients with renal transplants are glucocorticoids. Early detection and management of this complication is crucial to the progression and prevention of collapse of femoral heads. In the early diagnosis of avascular necrosis radionuclide bone scintigraphy provides an useful information on the aetiology of the painful hip before changes appear on $X$ ray examination. We present a case of proved glomerulonephritis taking glucocorticoids with difficulty in walking and pain around hip joints. A planar whole body and a SPECT Technetium 99-m (Tc99-m) bone scans were done which revealed avascular necrosis of both femoral heads. JMS 2012;15(1):61-64.

Key Words: Corticosteroids, avascular necrosis, glomerulonephritis, Tc99-m MDP Bone scan, SPECT, MRI

Cellular death of bone components due to interruption of the blood results in a condition called as Avascular necrosis.If the blood supply to the bone tissue is not restored it dies and the bone collapses. ${ }^{1}$ Avascular necrosis(AVN) involving bones of a joint often leads to destruction of the joint articular surfaces. Chemotherapy, alcoholisms, excessive steroid use, trauma, decompression sickness, vasculitis, arterial embolism thrombosis, damage from radiation, bisphosphonates (particularly the mandible), sickle cell anaemia and deep diving are the common risk factors implicated in the development of avascular necrosis of bone. ${ }^{2,3,4}$ In some cases it is idiopathic (no cause is found). Rheumatoid arthritis and lupus are also common causes of AVN. Glucocorticoids induced avascular necrosis of bone is

\section{Correspondence:}

Dr. ShoukatH Khan, M.D.

Head,

Department of Nuclear Medicine,

Sher-i-Kashmir Institute of Medical Sciences,Srinagar, J\&K, India-190011.

E-Mail:drshkhan199@yahoo.co.in sometimes found among patients with renal transplants, head injuries, systemic lupus eryhthematosis, ulcerative colitis, pemphigus, glomerulonephritis, Guillain-Barre syndrome etc. The femoral head is commonly involved although involvement of other sites like humeral head, femoral condyles, and ankles have been reported. ${ }^{5}$ A 40 year non alcoholic old man with renal biopsy proven minimal change glomerulonephritis on oral glucocorticoids with pain and restricted movements at both hip joints for a period of one and half years was referred for a Tc-99m MDP bone scan. Patient gave history of consuming, prednisolone $50 \mathrm{mg}$ daily for a period of one year prior to the present complaints. On planar Tc-99mMDP whole body bone scan and a subsequent SPECT features suggestive of AVN of both the hip joints were seen. A subsequent MRI of pelvis revealed hypointense lesions on T1 \& T2 weighted images in both the femoral heads. The case demonstrates the diagnostic usefulness of Tc-99m MDP bone scan and SPECT in patients suspected to have avascular bone necrosis due to steroid consumption. 


\section{Case report}

A 40 year old man with a renal biopsy proven minimal change glomerulonephritis taking $50 \mathrm{mg}$ of oral glucocorticoids (Prednisolone) daily for one year was referred to the Department of Nuclear Medicine, Sher-iKashmir institute of Medical Sciences Srinagar for a radionuclide bone scan. He complained of difficulty in walking and pain around both the hip joints. Personal history included an appendectomy done 25 years ago. The patient was a non alcoholic and there was no significant history of trauma causing skeletal injury. General examination was unremarkable except for an appendectomy scar on the abdomen. Local examination revealed painful limitation of movements in both the hip joints. There was an unmistakable waddle in his gait. Investigation profile revealed normal haemoglobin and blood counts. Renal function that included blood urea nitrogen $(22 \mathrm{mg} / \mathrm{dl})$ and creatinine $(1.2 \mathrm{mg} / \mathrm{dl})$ were with in normal limits. Serum calcium ( $9 \mathrm{mg} / \mathrm{dl})$, phosphorus $(3.4 \mathrm{mg} / \mathrm{dl})$, serum alkaline phosphatise(90U/l) were also within normal limits. A plain x-ray of pelvis (Figure 1) revealed irregularity of the subarticular bone in both the femoral heads with evidence of sclerosis. Anterior and posterior whole body Tc-99m MDP bone scan (Figure 2) revealed photopenic areas in both femoral heads with increased uptake in the surrounding superior-lateral aspects of the femoral heads. Both the kidneys were visualised. The planar Tc-99m MDP scan was followed by a Tc-99m MDP 360 degrees bone SPECT of hip region acquired in a step and

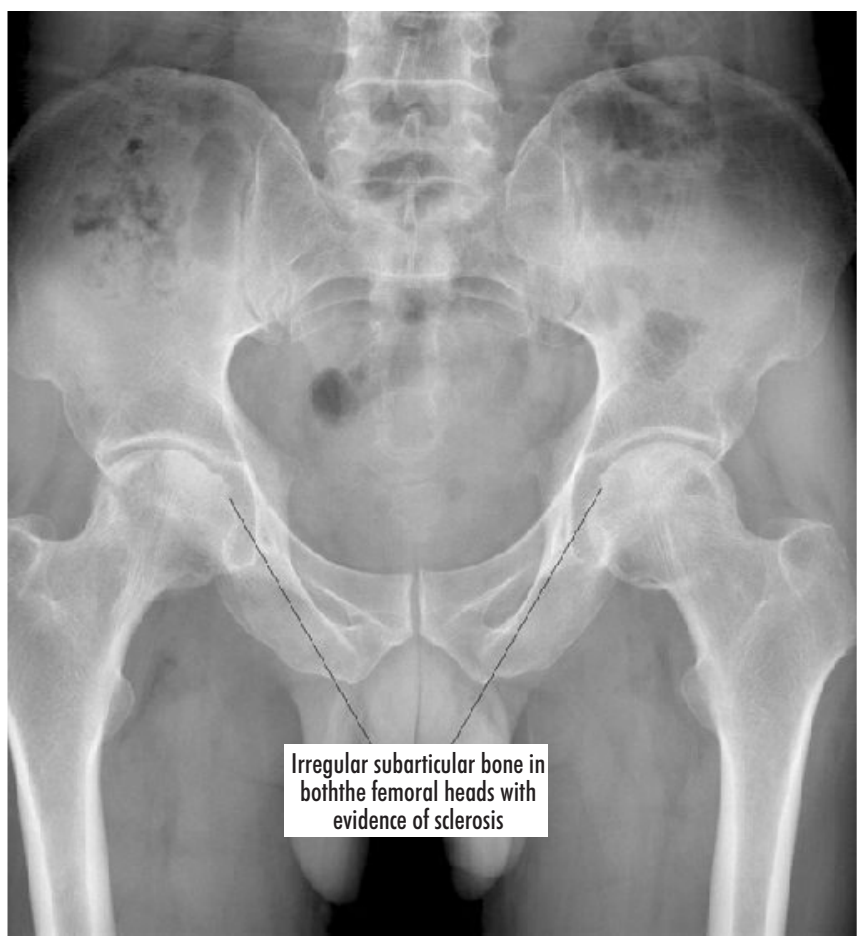

FIGURE 1. Plain X-RAY Pelvis (AP): Irregularity of the subarticular bone in both the femoral heads with evidence of sclerosis.

shoot mode in a noncircular rotation, $25 \mathrm{Sec}$ per projection with 5.6 degree rotation and a $128 \times 128$ matrix size. The
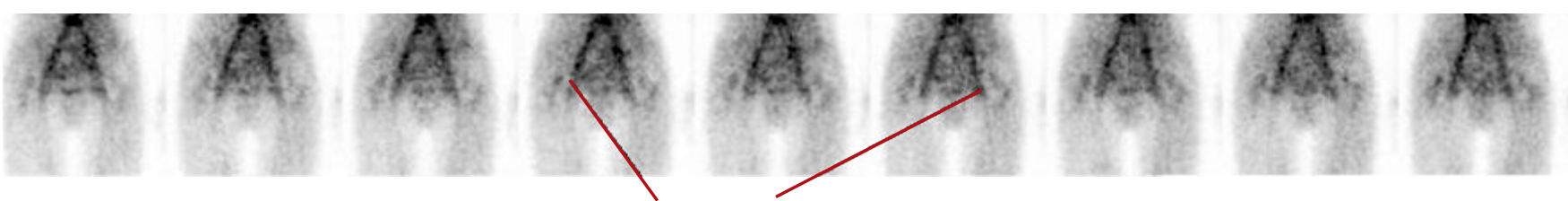

Early angiographic imaging showing increased blood flow to both the femoral heads (Ant images)

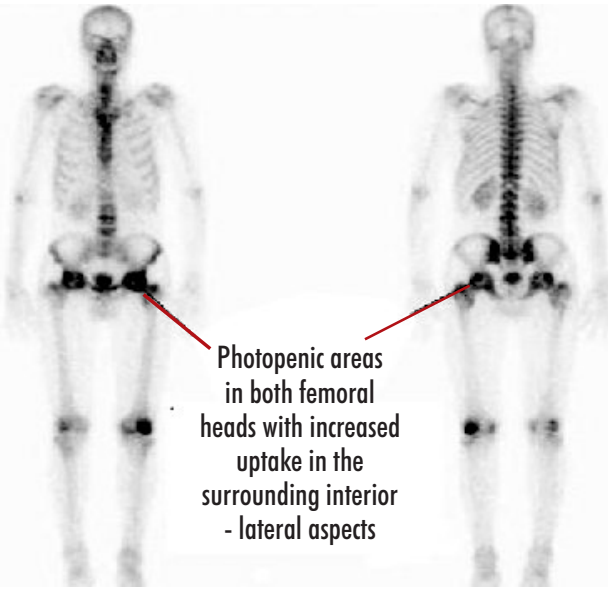

Anterior whole body Immoages Posterior whole body Images

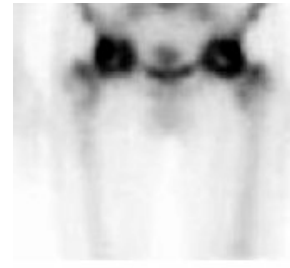

Anterior Static Images

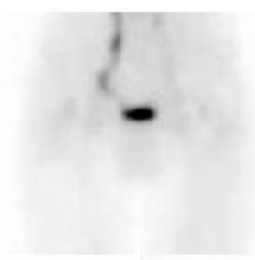

Posterior Static Images

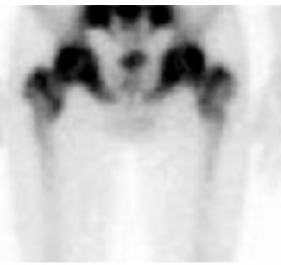

Anterior Images

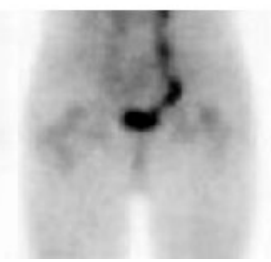

Posterior Image

FIGURE 2. TC-99m MDP 3-phase and whole body planar bone scan. Anterior and posterior whole body Tc-99m MDP bone scan (Figure-2) revealing photopenic areas in both femoral heads with increased uptake in the surrounding superior-lateral aspects of the femoral heads. 


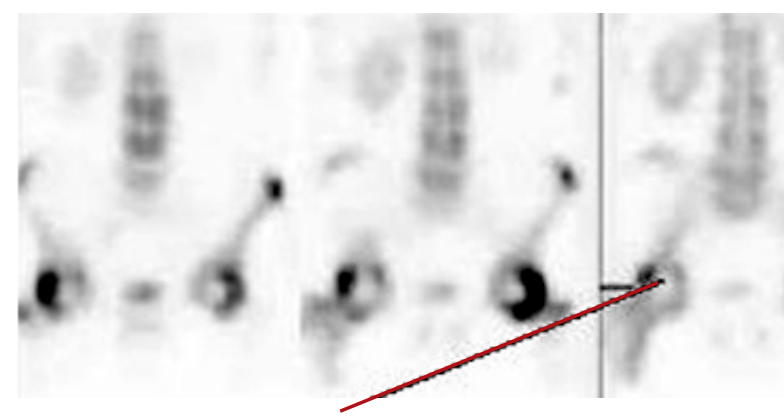

Rt. femoral head

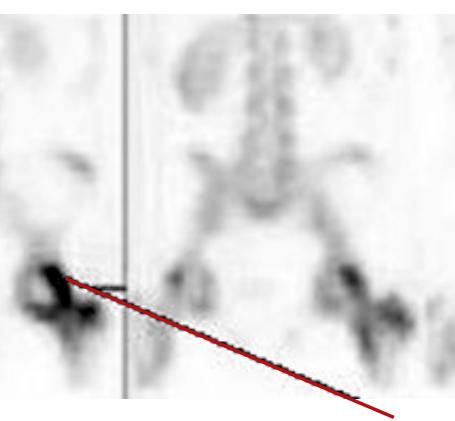

Lt. femoral head

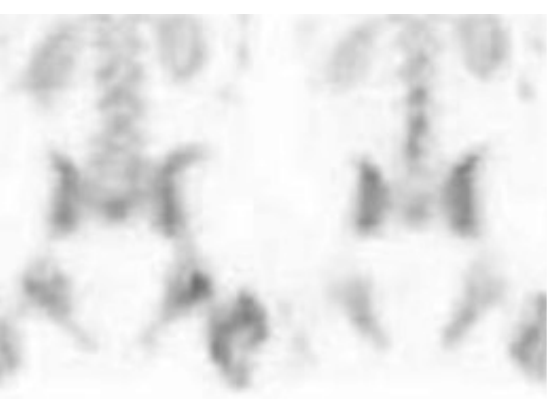

FIGURE 3: Coronal sections ofTC-99m MDP BONE SPECT. Type 2 defect of a photopenic area in both femoral heads with adjacent increased uptake consistent with type-2 bilateral femoral head avascular necrosis

SPECT images (Figure-3) were reconstructed using OSEM iterative reconstruction with a Gaussian filter (cut off 6). The select SPECT images revealed a type 2 defect of a photopenic area in both femoral heads with adjacent increased uptake consistent with type-2 bilateral femoral head avascular necrosis. A subsequent MRI on a 1.5-T scanner done after one week revealed the presence of low signal intensity in both femoral heads on T1 and T2 weighted images (Figures 4,5).

\section{Discussion}

Glucocorticoid induced avascular necrosis of bone is found in patients with renal transplants, lupus eryhthematosus, glomerulonephritis, peripheral neuritis, pemphigus, Guillain- Barre syndrome, head injuries and those given combination chemotherapy. ${ }^{6}$ The common reasons attributed to explain predisposition of patients taking glucocorticoid to avascular necrosis of bone are fat embolism, ${ }^{7}$ hypercoagubility, ${ }^{8}$ increased intraosseous pressure, and fat cell swelling. ${ }^{10}$ Early detection of this disorder is crucial to arrest its progression and prevent the collapse of femoral head ${ }^{11}$. An improved understanding of the pathophysiology of AVN has lead to early diagnosis and management of this disease entity. MRI and radionuclide bone imaging are more sensitive than plain radiography in the early stages of avascular bone necrosis particularly in circumstances where plain radiography is non conclusive or suspicion of avascular necrosis is high. ${ }^{12,13}$ SPECT has been reported to be more sensitive than MRI for detection of early (type 1) femoral head avascular necrosis. ${ }^{12}$ The present case highlights the utility of Tc-99m MDP bone Scintigraphy with SPECT imaging in the diagnosis of femoral head avascular necrosis among patients consuming gluco-

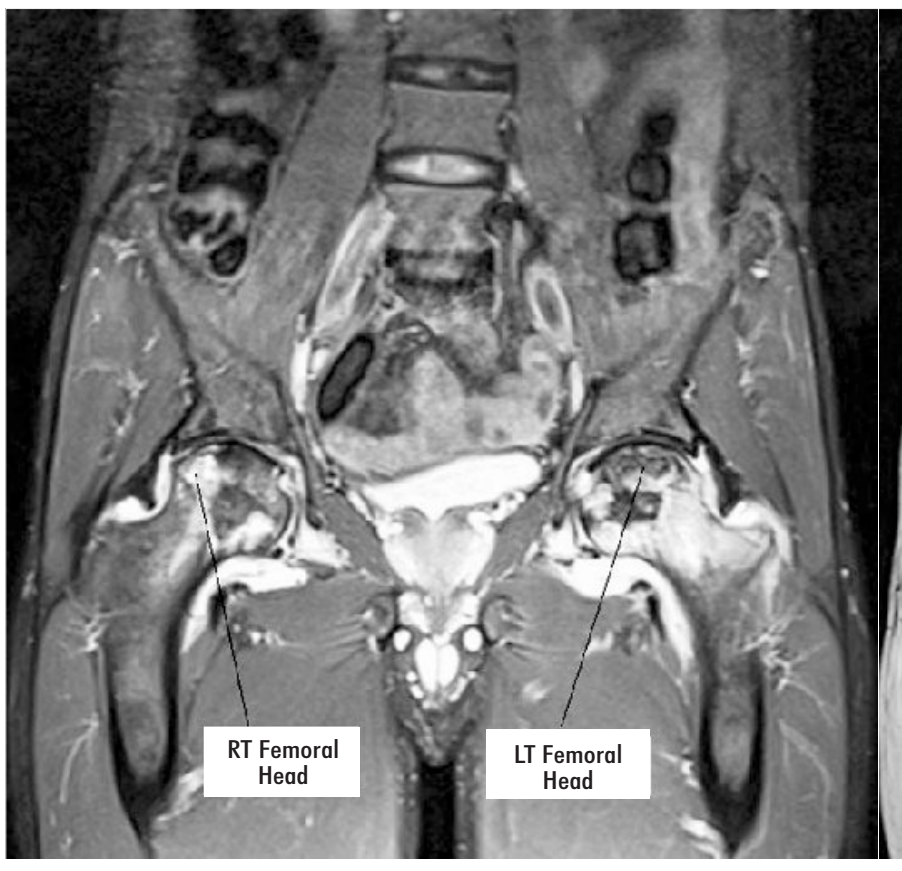

FIGURE 4. T1 weighted MRI(1.5-T scanner) Low signal intensity in both femoral heads on $\mathrm{Tl}$ weighted images

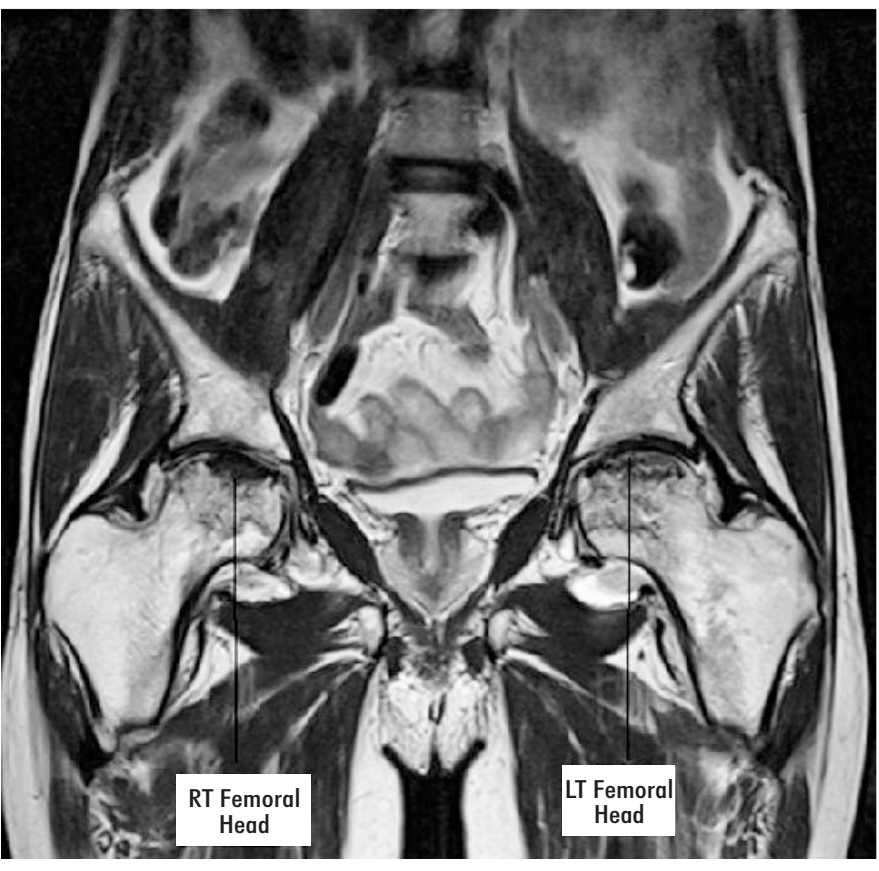

FIGURE 5. T2 weighted MRI. Low signal intensity in both femoral heads 
corticoids for various diseases and having pain or discomfort in the hip region.

\section{References}

1. Digiovanni CW, Patel A, Calfee R, Nickisch F . Osteonecrosis in the foot. The Journal of the American Academy of Orthopaedic Surgeons 2007;5(4):208-17.

2. Chao YC, Wang SJ, Chu HC, Chang WK, Hsieh TY. Investigation of alcohol metabolizing enzyme genes in Chinese alcoholics with avascular necrosis of hip joint, pancreatitis and cirrhosis of the liver. Alcohol and alcoholism 2003;38(5):431-36.

3. Juéry P. Avascular necrosis after a steroid injection. Canadian Medical Association Journal 2007;176(6):814-14.

4. Baksi DP. Treatment of post-traumatic avascular necrosis of the femoral head by multiple drilling and muscle-pedicle bone grafting. Preliminary report. The Journal of Bone and Joint Surgery. British volume 65(3): 26873.

5. Jacobs MA, Loeb PE, Hungerford DS. Core decompression of the distal femur for avascular necrosis of the knee. The Journal of Bone and Joint Surgery 1989). British volume 71(4):583-7.

6. Nixon JE. Early diagnosis and treatment of steroid induced avascular necrosis of bone. $\mathrm{Br}$ Med J (Clin Res Ed)1984;288(6419):741-44.
7. Jones JP. Alcoholism, hypercorticonism, fat embolism and osseous avascular necrosis In: Zinn W, ed. Idiopathic ischemic necrosis of the femoral head in adults. Stuttgart: Thieme, 197.

8. Boetthcher WG, Bonfiglio M, Hamilton HH, Sheets RF, Smith K. Non traumatic necrosis of femoral head. Part 1. Relation of altered hemostasis to etiology.J Bone Joint Surg 1970;52A:312-21.

9. Hungerford DS. Bone marrow pressure, venography and core decompression in ischemic necrosis of femoral head. In: The Hip society. The Hip: proceedings of the seventh open scientific meeting of the hip society. St Louis: C.V Mosby Co, 1979.

10. Wang JG, Sweet DE, Reger SI, Thompson RC. Fat cell changes as a mechanism of avascular necrosis of the femoral head in cortisone treated rabbits. J Bone Joint Surg. 1977; 59A:729-35.

11. Castro FP, Barrack RL. Core decompression and conservative treatment for avascular necrosis of femoral head: a meta analysis. Am J Orthop 2000;29:187-194.

12. Ryu JS, Kim JS, Moon DH, et al. Bone SPECT is more sensitive than MRI in the detection of early osteonecrosis of the femoral head after renal transplant. J Nucl Med 2002;43:1006-1011.

13. Siddiqui AR, Kopecky KK, Wellman HN, et al. Prospective study of magnetic resonance imaging and SPECT bone scan in renal allograft recipients: evidence for a self limited subclinical abnormality of the hip. $J$ Nucl Med 1993; 34:381-386. 